\title{
Polarimetry of the binary PDS $144^{\star}$
}

\author{
A. Pereyra ${ }^{1}$, C. V. Rodrigues ${ }^{2}$, and A. M. Magalhães ${ }^{3}$ \\ 1 Observatório Nacional, Rua General José Cristino 77, São Cristovão, 20921-400, Rio de Janeiro, Brazil \\ e-mail: pereyra@on.br \\ 2 Instituto Nacional de Pesquisas Espaciais/MCT, Avenida dos Astronautas 1758, São José dos Campos, SP, 12227-010, Brazil \\ 3 Departamento de Astronomia, IAG, Universidade de São Paulo, Rua do Matão 1226, São Paulo, SP, 05508-900, Brazil \\ Received 14 September 2011 / Accepted 12 December 2011
}

\section{ABSTRACT}

\begin{abstract}
Aims. Our goal is to study the circumstellar environment associated with each component of the wide intermediate-mass pre-main sequence binary system PDS 144 using broadband polarimetry.

Methods. We present near-infrared (NIR) linear polarimetric observations of PDS 144 gathered with the IAGPOL imaging polarimeter along with the CamIV infrared camera at the Observatório do Pico dos Dias (OPD). In addition, we re-analyzed OPD archive optical polarization to separate the binary and estimate the interstellar polarization using foreground stars.

Results. After discounting the interstellar component, we found that both stars of the binary system are intrinsically polarized. The polarization vectors at optical and NIR bands of both components are aligned with the local magnetic field and the jet axis. These findings indicate an interplay between the interstellar magnetic field and the formation of the binary system. We also found that the PDS $144 \mathrm{~N}$ is less polarized than its southern companion in the optical. However, in the NIR PDS $144 \mathrm{~N}$ is more polarized. Our polarization data can only be explained by high inclinations $\left(i \gtrsim 80^{\circ}\right)$ for the disks of both members. In particular, comparisons of our NIR data with young stellar objects disk models suggest predominantly small grains in the circumstellar environment of PDS 144N. In spite of the different grain types in each component, the infrared spectral indexes indicate a coeval system. We also found evidence of coplanarity between the disks.
\end{abstract}

Key words. circumstellar matter - polarization - stars: pre-main sequence - stars: individual: PDS 144

\section{Introduction}

PDS 144 is the optical counterpart of the infrared source IRAS 15462-2551 (Carballo et al. 1992), identified as a $5^{\prime \prime}$ binary system on the Pico dos Dias Survey (Gregorio-Hetem et al. 1992) with both components classified as Herbig Ae stars (Torres et al. 1995). Vieira et al. (2003) reported spectral types A2 IV and A5 V for the northern (PDS 144N) and southern (PDS 144S) members, respectively. They also detected $\mathrm{H} \alpha$ emission around both stars, probably associated with accretion disks, along with forbidden emission lines, which indicate outflow activity. This was confirmed by the detection of jets emanating from both components by Grady et al. (2009 and priv. comm.). Interestingly, PDS 144N is cited as the only Herbig Ae star with an optically thick edge-on disk spatially resolved (Perrin et al. 2006, hereafter P06). The two stars are assumed to be the components of a physical binary because they both are pre-main sequence objects, but their circumstellar material is different in composition (Schütz et al. 2009) and probably in geometry (P06). The distance of the PDS 144 system is uncertain, but considering the more accepted value (1 kpc), its separation of $~ 5400$ AU would indicate a really wide binary. The high galactic latitude $\left(+22^{\circ}\right)$ is also peculiar, locating this system well above the Galactic plane (360 pc, P06).

Polarimetry is a powerful tool for detecting asymmetries in the environment around young stellar objects (YSOs). This is especially important for non-resolved objects, where the technique provides a direct diagnostic. The scattering of light produced by

\footnotetext{
* Based on observations obtained at the Observatório do Pico dos Dias, LNA/MCT, Itajubá, Brazil.
}

gas or dust in outflows, jets, lobes, and/or disks produces polarization that can give us information about the geometry and physical properties of these environments. There are few models of integrated polarization with available grids to be compared with observations of YSOs. In particular, Fischer et al. (1996) presented near-infrared (NIR) polarization models with different distributions of circumstellar material and grain sizes. Complementarily, Robitaille et al. (2006) produced a sample of multiband integrated polarization models for YSOs with selected masses and evolution stages. In this sense, multicolor polarimetry can help to verify what type of dust population and geometry is present in a YSO environment.

The geometric configuration of the disks and orbital planes in young binaries is an important observable that a successful theory of binary formation - via fragmentation or capture - has to explain (Bate et al. 2000). Furthermore, the dynamical interaction between circumstellar disks determines their evolution and hence is relevant for the study of the proto-planetary environment. Indeed, binary formation models that include cloud fragmentation tend to produce aligned circumstellar disks. On the other hand, capture scenarios predict arbitrary disk alignments.

In this regard, the direction of the polarization position angle can be used to trace the projected YSO disks on the sky (Pereyra et al. 2009). Interestingly, polarization measurements suggest binary systems with aligned disks within $30^{\circ}$ of each other (Jensen et al. 2004; Monin et al. 2006). However, coplanarity between the binary orbit and aligned disks cannot be assumed in closer and wider systems (Prato \& Weinberger 2010).

In this work, we combine archive optical polarization measurements with new NIR data to study the PDS 144 system. The observations and data reduction are presented in Sect. 2. Our 
Table 1. Log of observations and polarimetry of the PDS 144 system.

\begin{tabular}{|c|c|c|c|c|c|c|c|c|c|}
\hline \multirow[b]{2}{*}{ Object } & \multirow[b]{2}{*}{ Filter } & \multirow[b]{2}{*}{$\operatorname{Mag}^{a}$} & \multirow[b]{2}{*}{ Date } & \multirow[b]{2}{*}{$\begin{array}{c}\text { Exposure }^{b} \\
\text { (s) }\end{array}$} & \multirow[b]{2}{*}{$\begin{array}{l}\text { Aperture } \\
\text { (") }\end{array}$} & \multicolumn{2}{|c|}{ Observed polarization } & \multicolumn{2}{|c|}{ Intrinsic polarization } \\
\hline & & & & & & $\begin{array}{c}P \\
(\%)\end{array}$ & $\begin{array}{l}\text { PA } \\
\left(^{\circ}\right)\end{array}$ & $\begin{array}{c}P \\
(\%)\end{array}$ & $\begin{array}{l}\text { PA } \\
\left(^{\circ}\right)\end{array}$ \\
\hline \multirow[t]{4}{*}{ PDS 144S } & $\bar{V}$ & 12.8 & 1999 Apr. 07 & 2400 & 3.1 & $5.43(0.10)$ & $119.2(0.5)$ & $4.72(0.10)$ & $119.7(0.6)$ \\
\hline & $H$ & 9.0 & 2010 Jun. 15 & 960 & $5.0^{d}$ & $2.61(0.09)$ & $113.5(1.0)$ & $2.37(0.09)$ & $113.2(1.1)$ \\
\hline & $H$ & & 2010 Jun. 17 & 1440 & $5.0^{d}$ & $2.84(0.05)$ & $119.0(0.5)$ & $2.61(0.05)$ & $119.3(0.6)$ \\
\hline & $H$ & & average $^{c}$ & & & & & $2.48(0.12)$ & $116.4(1.4)$ \\
\hline \multirow[t]{4}{*}{ PDS $144 \mathrm{~N}$} & $V$ & 14.2 & 1999 Apr. 07 & 2400 & 3.1 & $3.70(0.14)$ & $142.8(1.1)$ & $3.32(0.14)$ & $147.8(1.2)$ \\
\hline & $H$ & 11.4 & 2010 Jun. 15 & 960 & 2.0 & $4.25(0.22)$ & $134.1(1.5)$ & $4.06(0.22)$ & $135.1(1.6)$ \\
\hline & $H$ & & 2010 Jun. 17 & 1440 & 2.5 & $4.35(0.07)$ & $134.6(0.5)$ & $4.16(0.07)$ & $135.6(0.5)$ \\
\hline & $H$ & & average $^{c}$ & & & & & $4.11(0.03)$ & $135.4(0.2)$ \\
\hline
\end{tabular}

Notes. Errors in parenthesis. ${ }^{(a)}$ From SIMBAD. ${ }^{(b)}$ Total integration time at eight waveplate positions. ${ }^{(c)}$ Average polarization over 2010 Jun. 15 and 17. ${ }^{(d)}$ In spite of the large aperture, the polarization is not contaminated by the PDS $144 \mathrm{~N}$ light. The polarization level is constant (within the errors quoted) between aperture radii $2.5-5.0^{\prime \prime}$.

results, which include an estimate of the foreground and intrinsic polarization, are shown in Sect. 3. We discuss correlations with the ambient magnetic field and the jets position angle (Sect. 4.1). We used dust models available in the literature to provide a constraint on the geometry and grain properties of the circumstellar environment associated with each component (Sect. 4.2). In addition, the coevality (Sect. 4.3), using spectral indexes, and the coplanarity (Sect. 4.4) of this wide system are also analyzed. Our conclusions are drawn in Sect. 5.

\section{Observations}

The NIR observations for PDS 144 were performed on 2010 June 15 and 17 using the Boller \& Chivens $0.6 \mathrm{~m}$ telescope at the Observatório do Pico dos Dias (OPD), Brazil. We used the second-generation IAGPOL, the IAG imaging polarimeter (Magalhães et al. 1996), along with the CamIV infrared camera and an $H$ broadband filter. CamIV is based on a HAWAII detector of $1024 \times 1024$ pixels and $18.5 \mu /$ pixel that yields a plate scale of $0.5^{\prime \prime} /$ pixel at this telescope $\left(\mathrm{FOV}=8^{\prime} \times 8^{\prime}\right)$. This plate scale is enough to resolve the binary system considering its separation of $5^{\prime \prime}$. The typical seeing during the observations was $1.5^{\prime \prime}$. The retarder used by IAGPOL was an achromatic $\lambda / 2$-waveplate optimized for NIR bands. The analyzer was a calcite Savart plate, which produces two orthogonally polarized images from every target in the field.

The PDS 144 system was observed through two sequences of four waveplate positions (WP) separated by 22.5 . In each WP, 40 images were obtained, hence a total of $(2 \times 4 \times 40=) 320 \mathrm{im}-$ ages were gathered. Dithering was performed following a linear pattern (in declination) from one WP to the next and repeated in each sequence of four WP. The sky was computed from the mode of the sequence. After flat-field correction, sky subtraction, and registration of the images, the PCCDPACK package (Pereyra 2000) was used to compute the polarimetric parameters. Observation of polarized standards yielded the position angle correction to the equatorial system. Unpolarized standard stars were used to check the instrumental polarization. This was found to be lower than $0.2 \%$ and no correction was applied to the data.

\section{Results}

\subsection{Observed polarization}

The results for NIR polarization of the PDS 144 components are shown in Table 1. The proper photometric aperture radii were selected by avoiding overlap between the two components of the binary and trying to maximize the resulting polarization accuracy. This accuracy is calculated from the spread of the measurements at each WP with regard to the expected four-cosine curve. Perrin et al. (2006) presented NIR polarization maps of PDS 144N but without a positive polarization detection in PDS 144S. The results presented here are the first NIR broadband polarization data for the PDS 144 system, to the best of our knowledge.

The optical polarimetry at $V$ band was obtained from archive data from Rodrigues et al. (2009). These observations were also gathered at OPD with the same telescope in 1999 April using IAGPOL and an optical $\lambda / 2$-waveplate, along with a CCD camera. In this work, a large aperture $\left(8^{\prime \prime}\right)$ was used to compute the polarization of PDS 144. This aperture encircled the two components and an average level of $4.65 \%$ with $\mathrm{PA}^{1}=124^{\circ} .1$ was quoted. To disentangle the polarization of each component, and taking into account their separation of 5.4" (P06), we made a new reduction of these data using small apertures. The typical seeing in these observations was $2.2^{\prime \prime}$. Our results are shown in Table 1 .

\subsection{Intrinsic polarization}

In general, the observed polarization of a source is composed of an intrinsic component associated with the source itself along with a foreground polarization produced by interstellar dust grains between the source and the observer. The relative contribution of intrinsic and foreground components depends on the wavelength. Rodrigues et al. (2009) estimated the foreground polarization for PDS 144 using the weighted average of the polarization of field stars within a $10^{\prime} \times 10^{\prime}$ area around the source. They used objects with a polarization signal-to-noise ratio $\left(P / \sigma_{\mathrm{P}}\right)$ higher than 3 . We performed a new reduction with a higher object detection threshold to minimize the presence of artifacts. To compute the average, we used only objects with $P / \sigma_{\mathrm{P}}>5$ (24 objects). We obtained $P_{V}^{\text {for }}=0.72 \% \pm 0.02 \%$ with $\mathrm{PA}=116^{\circ} \pm 1^{\circ 2}$. Our foreground estimate is consistent with the value quoted by Rodrigues et al. (2009) for PDS 144. The optical polarization map of field stars around PDS 144 is shown in Fig. 1 (left, red lines). Interestingly, P06 obtained an extinction estimate toward PDS $144 \mathrm{~S}$ ( $A_{V}=1.1 \mathrm{mag}$ ) fitting the spectral energy distribution (SED). With this $A_{V}$, the maximum interstellar

\footnotetext{
1 PA: polarization position angle.

2 This error is $4^{\circ}$ if the standard deviation of the sample is used in the computation.
} 

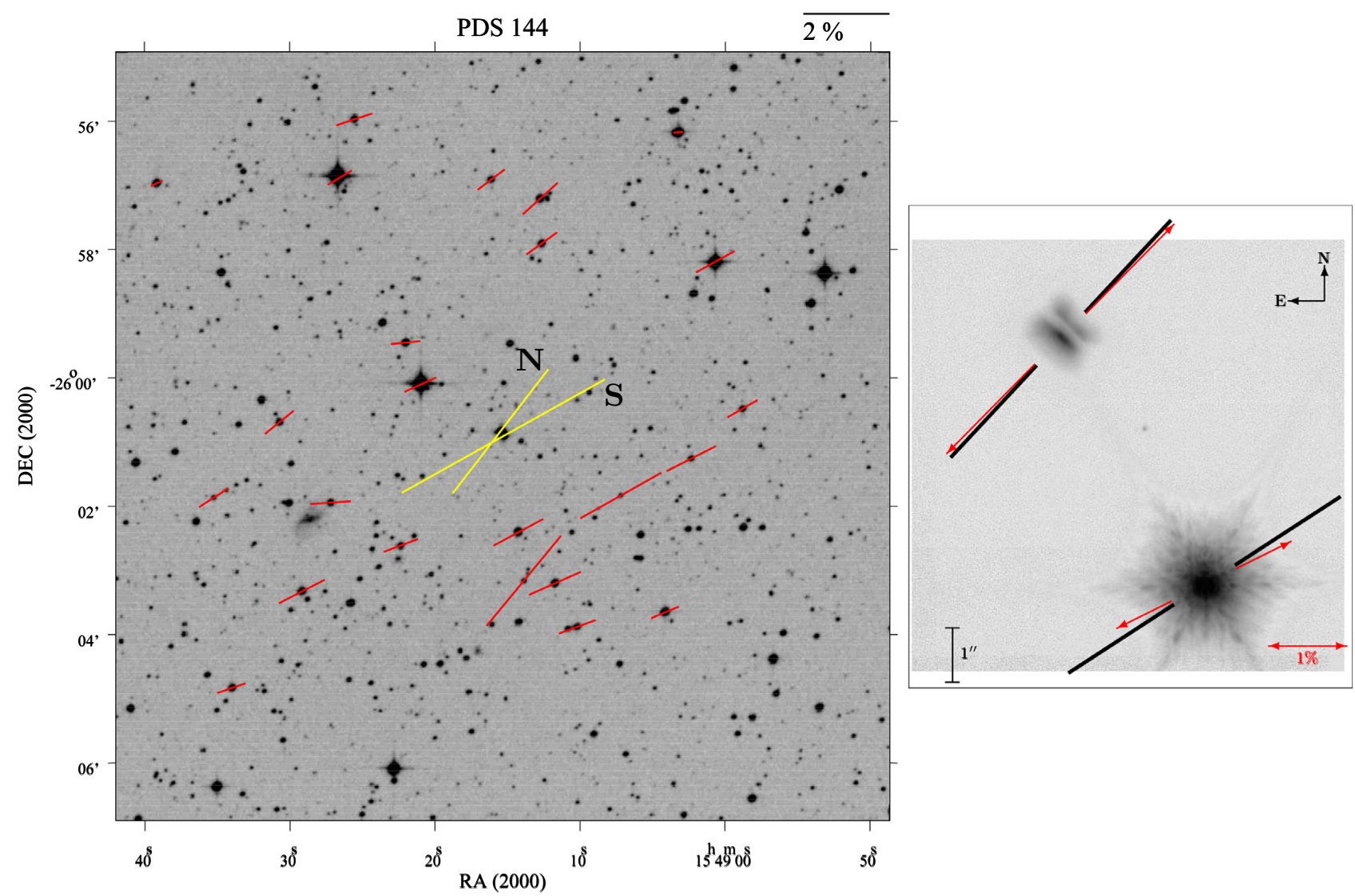

Fig. 1. Left: polarization map in the $V$ band of field stars around the PDS 144 system (red lines) plotted on a DSS2 Red image. The optical observed polarization for the binary components ( $\mathrm{N}$, north; $\mathrm{S}$, south) is also indicated (yellow lines). The polarization scale is shown in the top right. Right: NIR Keck AO imaging of PDS 144 (north and south) with the PDS 144N disk resolved (adapted from P06). The jet axis position angles for both objects are indicated by black thick lines: PDS $144 \mathrm{~N}=137^{\circ} \pm 3^{\circ}$; PDS $144 \mathrm{~S}=123^{\circ} \pm 2^{\circ}$ (Grady et al. 2009 and priv. comm.). The averaged intrinsic NIR polarization ( $H$ band, see Table 1$)$ is also indicated (red arrows). The polarization scale is indicated in the bottom right.

polarization (ISP) assuming an optimum polarization efficiency is $P_{\max }^{\mathrm{ISP}} \sim 3 \times A_{V}=3.3 \%$ (Serkowski et al. 1975). Compared with our foreground estimate, this result seems to indicate a weak polarization efficiency in this line of sight.

To estimate the foreground component in the $H$ band $(1.65 \mu \mathrm{m})$, we assumed that the foreground polarization can be represented by a standard Serkowski law, $P=P_{\max } \exp \left[-K \ln ^{2}\left(\lambda_{\max } / \lambda\right)\right]$ (Serkowski et al. 1975), with the maximum polarization, $P_{\max }$, which we assumed to occur at $\lambda_{\max }=0.55 \mu \mathrm{m}$ (it means, $P_{\max }=P_{V}^{\text {for }}$ ). Then, we extrapolated the Serkowski relation to NIR wavelengths and obtained the proper foreground polarization in the $H$ band. The resulting foreground polarization was $P_{H}^{\text {for }}=0.24 \%$. We assumed that the foreground PA is constant with wavelength.

For each component of the PDS 144 binary system, we computed the intrinsic polarization in the $V$ and $H$ bands using the Stokes parameters ${ }^{3}$, as follows:

$Q_{\text {int }}=Q_{\text {obs }}-Q_{\text {for }} \quad$ and $\quad U_{\text {int }}=U_{\text {obs }}-U_{\text {for }}$.

The intrinsic polarization $\left(P_{\text {int }}\right)$ and its polarization angle $\left(\mathrm{PA}_{\text {int }}\right)$ are obtained from

$P_{\text {int }}=\left(Q_{\text {int }}^{2}+U_{\text {int }}^{2}\right)^{1 / 2}$ and $\mathrm{PA}_{\text {int }}=\frac{1}{2} \tan ^{-1}\left(U_{\text {int }} / Q_{\text {int }}\right)$.

The results are indicated in Table 1 . They confirm that the intrinsic polarization dominates both components of PDS 144.

\footnotetext{
${ }^{3} Q=P \cos (2 \times \mathrm{PA}) ; U=P \sin (2 \times \mathrm{PA})$.
}

\section{Discussion}

\subsection{Alignment with the ambient magnetic field and jet axes}

The optical polarization map (Fig. 1, left) shows that the observed polarizations for both components of the PDS 144 system (yellow lines) are higher than the polarization of field stars. As mentioned above, this is a first indication that a large part of the observed polarization is produced in the circumstellar matter of PDS 144N and PDS 144S. The computed values (Table 1) confirm this, with the ISP being negligible (Fig. 2a), especially at NIR bands. Interestingly, at optical bands the intrinsic polarization of both components is aligned (within $30^{\circ}$, Fig. $2 \mathrm{~b}$ ) with the local magnetic field traced by the field stars. The alignment is stronger in the NIR (within $20^{\circ}$ ), with the PDS $144 \mathrm{~S}$ polarization position angle practically coincident with that of the ISP. This is indeed indicative that the initial collapse occurred in the direction parallel to the interstellar magnetic field for both stars. As long as this result does not depend on the stars being in a binary system, this finding is consistent with the conclusion from Rodrigues et al. (2009) of an ambient (i.e., large-scale) magnetic field that defines the shape of the circumstellar material around intermediate-mass young stars. Interestingly, a similar alignment was found between optical polarization and the ISP by Monin et al. (2006) in two thirds of a sample of T Tauri binaries. Therefore, this corroborates the evidence of an interplay between the interstellar magnetic field and the formation of lowand intermediate-mass binary systems. 


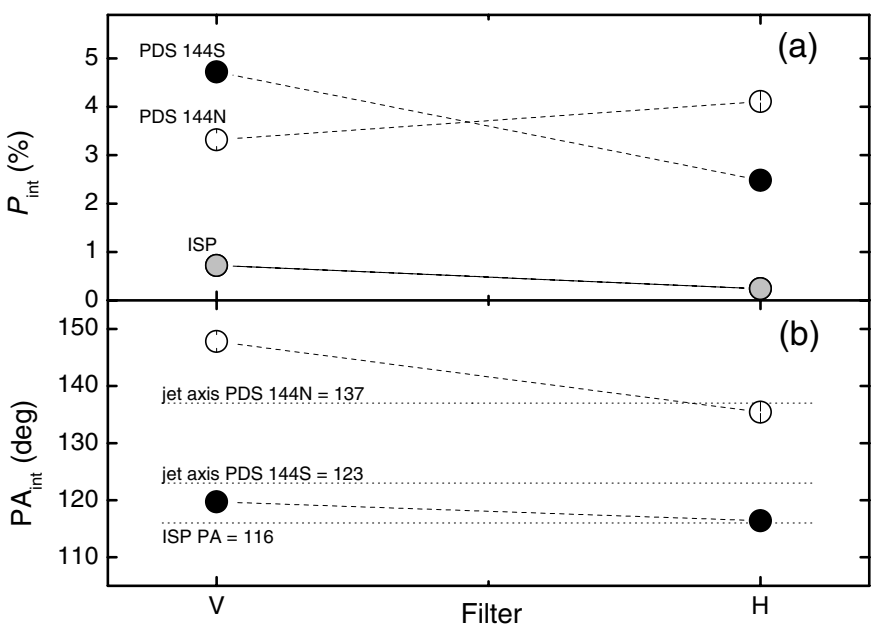

Fig. 2. Comparison between the optical and the averaged NIR intrinsic polarization for the PDS 144 system. a) Polarization level of PDS 144S (black dots) and PDS 144N (white dots). The interstellar polarization is also shown (gray dots). b) Polarization position angles for the system. The jet axes are also indicated (dotted lines) along with the ISP PA.

When disks are unresolved, collimated jets or outflows can be used to infer the disk orientation, assuming they are being ejected perpendicularly to the disks (Pereyra et al. 2009). Evidence of bipolar outflows was detected in both components of PDS 144 by Grady et al. (2009 and priv. comm.). The direction for the approaching jets is $137^{\circ} \pm 3^{\circ}$ and $303^{\circ} \pm 2^{\circ}$ for PDS $144 \mathrm{~N}$ and $144 \mathrm{~S}$, respectively. We found a remarkable agreement between the position angles of the intrinsic NIR polarization and the jet axes (Fig. 1, right). We observed an alignment within $2^{\circ}$ for PDS $144 \mathrm{~N}$, and within $10^{\circ}$ for PDS $144 \mathrm{~S}$ (Fig. 2b). The resolved disk in PDS $144 \mathrm{~N}$ has a $\mathrm{PA}_{\text {disk }} \sim 46^{\circ}(\mathrm{P} 06)$ that is rigorously perpendicular to the jet and NIR polarization. On the other hand, the PA for the unresolved disk of PDS 144S can be inferred using the perpendicularity between jets and disks $\left(\mathrm{PA}_{\text {disk }}=\mathrm{PA}_{\text {jet }}-90^{\circ}=33^{\circ}\right)$. Moreover, our intrinsic polarization PA for PDS 144S remains approximately constant at optical and NIR bands, and can also be used to confirm the precise direction of the PDS $144 \mathrm{~S}$ disk plane projected on the sky $\left(\mathrm{PA}_{\text {disk }}\right.$ between $26^{\circ}$ and $30^{\circ}$ ). Therefore, both disks in the PDS 144 system seem to be aligned within no more than $20^{\circ}$ (see Sect. 4.4 for a more detailed discussion).

\subsection{Circumstellar material around PDS $144 N$ and PDS $144 S$}

The detection of intrinsic polarization in both components of PDS 144 indicates that the two stars have asymmetric circumstellar material. The polarization degrees are similar in both stars, but the wavelength dependences are definitely different: the polarization of PDS 144S decreases from $V$ to $H$ band; the inverse occurs for PDS $144 \mathrm{~N}$. It has been assumed that the polarization of both components remains unchanged during the 11 years between the optical and NIR measurement. This assumption cannot be true, however, because the polarization of the majority of the Herbig Ae/Be stars is variable (Bastien 1988; Grinin 1994). In this section, we discuss these results considering the dust models available in the literature.

The spectral distribution of flux and polarization of YSOs has been studied in many works (see, for example, Brown \& McLean 1977; Bastien \& Ménard 1990; Fischer et al. 1996; Robitaille et al. 2006). They show that the observed SED and polarization spectra are very dependent on

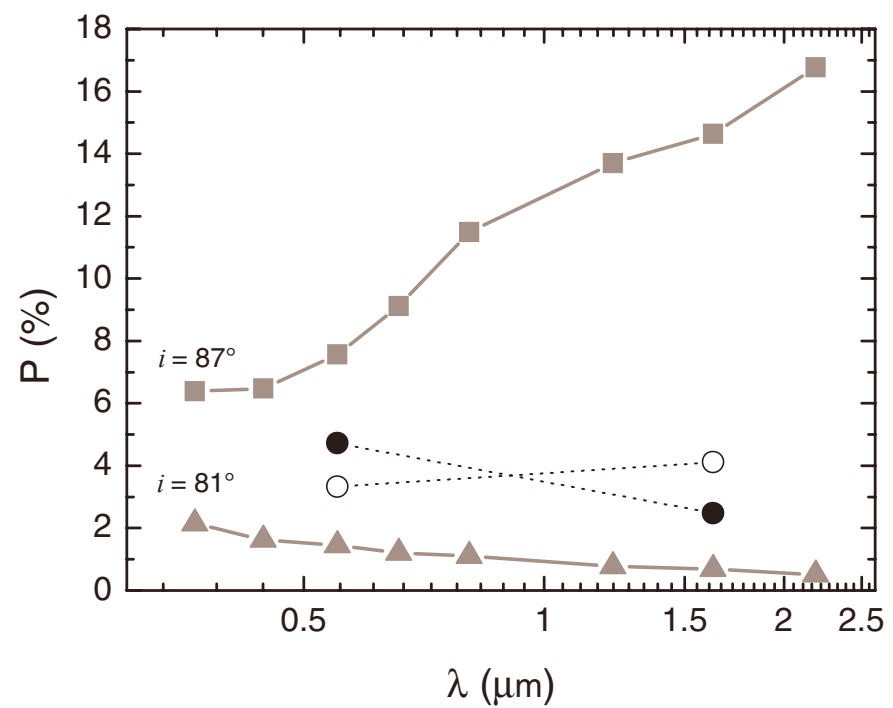

Fig. 3. Comparison of optical $(V)$ and NIR $(H)$ polarimetry of the binary PDS 144 (black dots: PDS 144S; and, white dots: PDS 144N) with the polarization YSO models from Robitaille et al. (2006). Taking into account the extreme edge-on configuration for each component of the binary, we show only the polarization wavelength dependence for models with two inclinations $\left(i=81^{\circ}\right.$, gray triangles; and, $i=87^{\circ}$, gray squares) and considering the stage II for a central source of $2 M_{\odot}$ (Fig. 9e from Robitaille et al. 2006, with positive polarizations perpendicular to the disk plane - as in the PDS 144 system).

stellar and circumstellar properties and the observer's line-ofsight. In general, these models suggest that if the polarization PA is parallel to the disk plane (or perpendicular to the jet axis), the disk will be inevitably optically thick. In turn, a polarization PA perpendicular to the disk plane cannot break the degeneracy between optically thin or thick disks, and additional modeling of the observed polarization level is needed. This is the case for both components of the PDS 144 system.

Fischer et al. (1996) produced models to explain the polarization pattern of the circumstellar environment around YSOs using different particle sizes. When small particles are used, their models predict high NIR polarization levels and a decreasing polarization wavelength dependence. In turn, large particles produce more modest polarizations along with an increasing polarization wavelength dependence. However, variations of the disk inclination in extreme edge-on configurations are also able to explain different polarization wavelength dependencies for YSOs (Robitaille et al. 2006).

Taking into account the above, we initially studied the circumstellar environment of PDS 144 components using the grid of YSO models with polarization wavelength dependence from Robitaille et al. (2006, Fig. 9). The circumstellar geometry consists of a rotationally flattened infalling envelope, bipolar cavities and a flared accretion disk. The grain properties are fixed in their models: they used grain sizes slightly larger than the ISM size distribution, and in the denser portion of the disk the grains are larger than $50 \mu \mathrm{m}$. Using their grid, our intrinsic optical and NIR polarizations for the PDS 144 system (both perpendicular to the disk planes, as shown in Fig. 1) are consistent with high inclinations $^{4}\left(i>81^{\circ}\right)$ of stage II YSOs with $\sim 2 M_{\odot}$ (see Fig. 3). This mass is representative of the spectral type associated with both components (A2-A5, Vieira et al. 2003). Interestingly, the

${ }^{4}$ In the particular case of PDS $144 \mathrm{~N}$, our result is also consistent with the inclination obtained by P06 $\left(i=83^{\circ}\right)$ modeling the shape of the observed resolved disk. 
above models show that in high inclinations, a variation of few degrees $\left(i \sim 87^{\circ}\right.$, in Fig. 3) can change the polarization value abruptly. Furthermore, there is an indication that the wavelength dependence also changes with inclination. This can explain the polarimetric observations of the PDS 144 system, where the polarization slopes have different behaviors. In PDS 144S, the polarization decreases from optical at NIR band, and the opposite is evident in PDS 144N. Additional multicolor polarimetry measurements would be very useful to better understand the grain size distribution in the PDS 144 system.

Another possible explanation for a change in the polarization slope can be achieved if the grain sizes are different in PDS 144N and 144S. In particular, Fischer et al. (1996) modeled the NIR polarization from YSOs using different recipes of circumstellar matter distribution and grain sizes. To facilitate the comparison, these models can be explored using the additional constraint of high inclinations $\left(i \gtrsim 80^{\circ}\right)$ for both disks in the PDS 144 system. Basically, these simulations include a circumstellar environment composed of a disk, a bipolar outflow and a cloud core with two different grain models tested (small ${ }^{5}$ and large grains). Several configurations of disk models ${ }^{6}$ including massive disks (TO, LA, RB, SL models) and a massless Keplerian disk (SH model) were considered in trying to reproduce evolutionary stages. These simulations used a low-mass central star ( $\left.T_{\text {eff }}=5000 \mathrm{~K}, L=10 L_{\odot}\right)$ and caution must be taken when comparing with intermediate-mass stars, as in the PDS 144 system. However, these models confirm that the youngest objects with powerful mass-outflows and "very" optically thick disks show a high degree of polarization parallel to the disk plane (TO disk). Earliest T Tauri phase shows low degrees of polarization (LA and RB disks with large grains) and normal T Tauri stars display little polarization perpendicular to the disk plane in a Keplerian and optically thin disk (SH disk with larger particles).

Figure 4 shows the spatially unresolved models from Fischer et al. (1996), which can explain our $H$-band polarimetry for the PDS 144 system. Considering the high inclination of both binary components, we selected only models that match the PDS 144 polarization levels for $i>80^{\circ}$. Only two models can reproduce our measurements for PDS 144N and four models for PDS 144S. All these models correspond to polarizations perpendicular to the plane disk (or negative sign in Figs. 9 and 10 from Fischer et al. 1996), which are consistent with our observations. Hence, the "very" massive disks (or TO models) and massive disks with high midplane densities were totally discarded for both components of PDS 144.

It is interesting to note that PDS $144 \mathrm{~N}$ can only be fitted by earlier YSOs (LA and RB models) with small grains. Models with large grains cannot explain the high polarization level observed in the northern component. On the other hand, PDS 144S, with a low polarization, can be reproduced by later YSOs with small grains (SL and SH models) or a combination of earlier (LA) or later YSOs (SH) with large grains. Interestingly, these results are consistent with the mid-IR spectroscopy at $N$-band of the PDS 144 system by Schütz et al. (2009). The PDS $144 \mathrm{~N}$ spectrum has prominent PAH bands (suggesting very small grains) in a flared disk without any silicate emission. In turn, the PDS $144 \mathrm{~S}$ has mainly large amorphous silicate grains but without evidence of processed dust.

However, the models from Fischer et al. (1996), discussed above, cannot explain the polarization reversal wavelength

\footnotetext{
5 Similar to grains in the diffuse ISM.

6 TO: Toomre (1982); LA: Larson (1984); RB and SL: Lazareff et al. (1990); and SH: Shakura \& Sunyaev (1973).
}

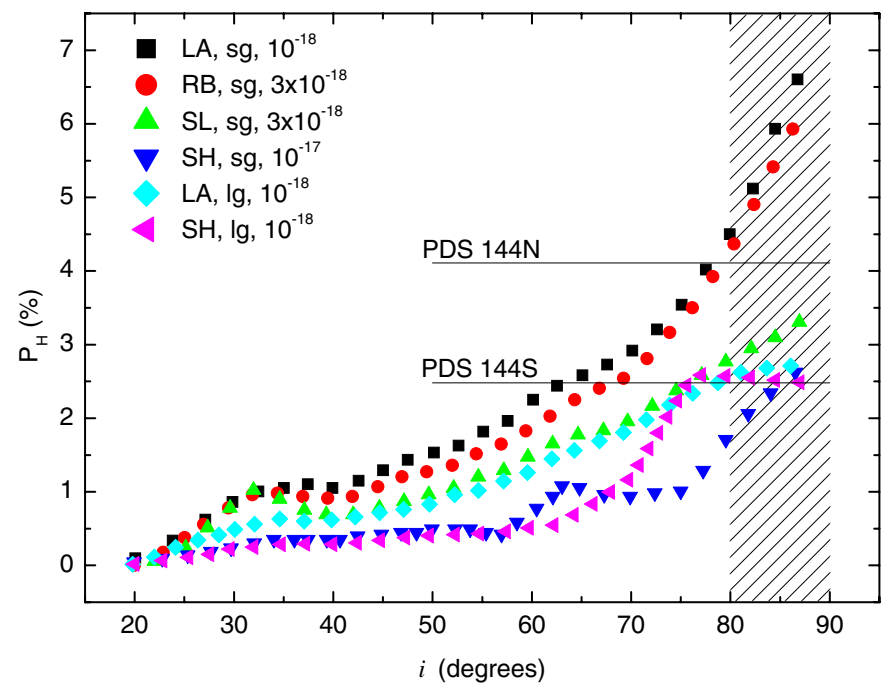

Fig. 4. Comparison of our intrinsic NIR polarimetry (Table 1) of the binary PDS 144 (horizontal lines) with selected models (symbols) of YSOs from Fischer et al. (1996), as a function of the inclination. All models shown here correspond to polarizations perpendicular to the plane disk (negative sign in Figs. 9 and 10 from Fischer et al. 1996). The models include different massive accretion disks (LA, RB and SL types) and a massless Keplerian disk (SH type); two families of grains (small grains: $s g$, and large grains: $l g$ ); and three values for the midplane density $\left(1,3,10 \times 10^{-18} \mathrm{~g} \mathrm{~cm}^{-3}\right)$. The shadow region indicates the high inclinations associated with the PDS 144 system.

dependence observed in the PDS 144 components. These models predict that small grains will have positive slopes at NIR bands, and the opposite for large grains. In particular, the small grain models, which best fit the PDS 144N $H$-band polarization, are not consistent with the positive slope observed between $V$ and $H$ bands. In this sense, the observed polarization reversal seems better explained by the high-inclination dependence (Robitaille et al. 2006), with PDS $144 \mathrm{~N}$ being more inclined that its companion and showing the enhanced polarization at $H$ band.

In summary, the models seem to indicate a possible common evolutionary stage for both components but with important differences in their dust grain populations. Indeed, P06, who analyzed the SED of the binary, also pointed out differences between the components, with PDS 144N having a greater amount of cool dust at larger radii than does PDS 144S.

\subsection{Coevality}

We can use the NIR and mid-IR spectral indexes (Lada 1987; Greene et al. 1994) to verify if both components are in a similar evolutionary phase. In this sense, using the 2MASS and mid-IR photometry (the last one from P06), we computed $\alpha(K, 24)=\log \left[\lambda_{24} F_{K} /\left(\lambda_{K} F_{24}\right)\right] / \log \left(\lambda_{K} / \lambda_{24}\right)$, the spectral index between the 2MASS $K$ band and the Spitzer MIPS $23.7 \mu \mathrm{m}$ flux for each component. This index is similar to $\alpha(K, 25)$ using IRAS fluxes, and was used by Pereyra et al. (2009) to analyze correlations between the relative direction of the disk plane and the polarization PA in a sample of YSOs. For PDS 144, IRAS fluxes do not resolve the system and only the MIPS bands separate the components properly. The $\alpha(K, 24)$ spectral indexes are +0.36 and -0.15 for PDS $144 \mathrm{~N}$ and $144 \mathrm{~S}$, respectively. Considering the classification of Lada (1987), PDS 144N will be a class I YSO $(0<\alpha \leq 3)$ and PDS 144 S, a class II $(-2 \leq \alpha \leq 0)$. This seems inconsistent if we assume that 
the system is coeval and both components have similar spectral types (A2 IV and A5 V for PDS 144N and 144S, respectively; Vieira et al. 2003).

However, SED fitting by P06 using optical, NIR and mid-IR colors needed interstellar extinction corrections (see Sect. 3.2) of $A_{V}=3$ and $1.1 \mathrm{mag}$ for PDS $144 \mathrm{~N}$ and $144 \mathrm{~S}$, respectively. With this correction applied to the 2MASS $K$ band (following $A_{V} / A_{K}=8.8$, Cardelli et al. 1989), the new spectral indexes are +0.23 and -0.20 for PDS $144 \mathrm{~N}$ and 144 S, respectively. Interestingly, if we consider the more recent classification from Greene et al. (1994), both components of the system will be included in the flat-spectrum type $(-0.3 \leq \alpha \leq 0.3)$, and therefore the coeval binary could be restored.

\subsection{Coplanarity}

More information can be extracted comparing the polarization PA between each component of the system. Using again the NIR polarization PA, we found that the north and south components of PDS 144 are aligned within $15^{\circ}$. This implies that the projected disk planes of both components are also aligned and therefore coplanarity between them is possible. This is reinforced considering the similar and high inclinations $\left(i \gtrsim 80^{\circ}\right)$ for both components discussed above. In addition, these inclinations along with the opposite configurations north-south for the approaching jets in each component also favors the alignment within $20^{\circ}$. If this is correct, the fragmentation scenario of binary formation is more likely in the PDS 144 system. Interestingly, considering the correlation between distance and alignment, using polarization data of binary systems already published (Fig. 1 in Jensen et al. 2004; and Fig. 11 in Monin et al. 2006), PDS 144 would be the aligned system with the higher projected binary separation ( 5400 AU).

On the other hand, alignment of the circumstellar disks does not necessarily imply coplanarity of the binary orbital plane with that of the aligned disks (Prato \& Weinberger 2010). This, for example, is the case of aligned disks with their disk planes rigorously perpendicular to the orbital plane. However, the PA of the binary separation in PDS 144 is $28^{\circ} .7$ (P06), which compares well with the $\mathrm{PA}_{\text {disk }}$ of both components (see Sect. 4.1). Only if we consider a binary orbital plane perpendicular to the sky, can we suppose coplanarity between the binary orbital plane and the disks. Therefore, coplanarity between the disks for PDS 144 system is very likely but more studies must be made to detect coplanarity with the binary orbital plane.

\section{Conclusions}

We have presented optical and near-infrared broadband polarimetry of the binary system PDS 144 . The intrinsic polarization in both bands reveals a strong alignment with the ambient magnetic field mapped using polarization of field stars. This finding confirms a really significant magnetic support for this young intermediate-mass system. Our results suggest that the original collapse, which formed both stars, happened in the direction parallel to the interstellar magnetic lines, as observed in other similar Herbig Ae/Be stars.

Comparing the optical and NIR polarizations, opposite polarization wavelength dependence is observed, with PDS $144 \mathrm{~N}$ being more polarized at NIR bands than its souther companion. When we compared our data with models available in the literature, high inclinations $\left(i \gtrsim 80^{\circ}\right)$ were needed to reproduce the polarization observed in both components. Interestingly, the high NIR polarization of PDS $144 \mathrm{~N}$ only can be explained by earlier YSOs with a distribution of grains of small sizes similar to those of the diffuse ISM. On the other hand, the lower polarization of PDS 144 S yields more possibilities to the YSOs models that prefer later YSOs with small grains or a combination of earlier and later YSOs, but with large grains. Assuming similar ages for our system, this seems to indicate significant differences of the grain populations of each component. We checked the coevality of the binary and were able to confirm it using infrared spectral indexes, according to which both components belong to flat-spectrum type.

Finally, we found evidence of coplanarity between the disk planes. Considering the extreme binary separation of PDS 144, this must be one of the few wide binary systems reported without misalignment. In this context, the scenario of fragmentation seems to the favored one for PDS 144.

Acknowledgements. A.P. thanks CNPq (DTI grant 382.585/07-03 associated with the PCI/MCT/ON program). A.M.M. acknowledges support from FAPESP (grant 01/12589-1) and CNPq. C.V.R. thanks CNPq (grant 308005/2009-0).

\section{References}

Bastien, P. 1988, in Polarized Radiation of Circumstellar Origin, ed. G. V Coyne, A. M. Magalhães, A. F. J. Moffat, R. E. Schulte-Ladbeck, S. Tapia, D. T. Wickramasinghe, Vatican Observatory, 541

Bastien, P., \& Ménard, F. 1990, ApJ, 364, 232

Bate, M. R., Bonnell, I. A., Clarke, C. J., et al. 2000, MNRAS, 317, 773

Brown, J. C., \& McLean, I. S. 1977, A\&A, 57, 141

Carballo, R., Wesselius, P. R., \& Whittet, D. C. B. 1992, A\&A, 262, 106

Cardelli, J. A., Clayton, G. C., \& Mathis, J. S. 1989, ApJ, 345, 245

Fischer, O., Henning, Th., \& Yorke, H. W. 1996, A\&A, 308, 863

Grady, C. A., Harding, M., Bonfield, D., et al. 2009, BAAS, 41, 224

Gregorio-Hetem, J., Lepine, J. R. D., Quast, G. R., Torres, C. A. O., \& de La Reza, R. 1992, AJ, 103, 549

Greene, T. P., Wilking, B. A., André, P., Young, E. T., \& Lada, C. J. 1994, ApJ, 434,614

Grinin, V. P. 1994, in The Nature and Evolutionary Status of Herbig Ae/Be Stars, ed. P. S. The, M. R. Perez, \& E. P. J. Van den Heuvel (San Francisco: ASP), ASP Conf. Ser., 62, 63

Jensen, E. L. N., Mathieu, R. D., Donar, A. X., \& Dullighan, A. 2004, ApJ, 600, 789

Lada, C. J. 1987, in Star Forming Regions, ed. M. Peimbert, M., \& J. Jugaku (Dordrecht: Reidel), IAU Symp., 115, 1

Larson, R. B. 1984, MNRAS, 206, 197

Lazareff, B., Pudritz, R. E., \& Monin, J.-L. 1990, ApJ, 358, 170

Magalhães, A. M., Rodrigues, C. V., Margoniner, V. E., Pereyra, A., \& Heathcote, S. 1996, ASP Conf. Ser. 97, ed. W. G. Roberge, \& D. C. B. Whittet, 118

Monin, J.-L., Ménard, F., \& Peretto, N. 2006, A\&A, 446, 201

Pereyra, A. 2000, Ph.D. Thesis, Instituto Astronômico e Geofísico, Universidade de São Paulo

Pereyra, A., Girart, J. M., Magalhães, A. M., Rodrigues, C. V., \& de Araújo, F. X. 2009, A\&A, 501, 595

Perrin, M. D., Duchêne, G., Kalas, P, \& Graham, J. R. 2006, ApJ, 645, 1272, P06

Prato, L., \& Weinberger, A. J. 2010, in Planets in Binary Star Systems (Astrophysics and Space Science Library, Springer Science+Business Media B.V.), 366, 1

Robitaille, T. P., Whitney, B. A., Indebetouw, R., Wood, K., \& Denzmore, P. 2006, ApJS, 167, 256

Rodrigues, C. V., Sartori, M. J., Gregorio-Hetem, J., \& Magalhães, A. M. 2009, ApJ, 698, 2031

Serkowski, K., Mathewson, D. S., \& Ford, V. L. 1975, ApJ, 196, 261

Shakura, N. I., \& Sunyaev, R. A. 1973, A\&A, 24, 337

Schütz, O., Meeus, G., Sterzik, M. F., \& Peeters, E. 2009, A\&A, 507, 261

Toomre, A. 1982, ApJ, 259, 535

Torres, C. A. O., Quast, G., de la Reza, R., Gregorio-Hetem, J., \& Lepine, J. R. D. 1995, AJ, 109, 2146

Vieira, S. L. A., Corradi, W. J. B., Alencar, S. H. P., et al. 2003, AJ, 126, 2971 